Historic, Archive Document

Do not assume content reflects current scientific knowledge, policies, or practices. 



\section{CLOSING OUT LIST}

$0 \mathrm{~F}$

\section{Gladioli, Dahlias and Iris}

Just a few odds and ends left, grown from bulblets and small size planting stock. L means $I$ in. up, mostly up. Some kinds are slow growers from bulblets, but bloom as well. M, $3 / 4$ to 1 inch. $\mathrm{S}, 1 / 2$ down. Blooming sizes are large enough to bloom and will produce large flowers and plenty of bulblets. Bulblets of all kinds sold from 1 to 100 at cheap rates. I have over 100 kinds in too small quantities to list. Send list of wants or send $\$ 1.00$ to $\$ 5.00$ and I will make up a collection of choice kinds at very low prices. 25 percent holds the order until March 1 st. Those marked * I will sell 6 at price of $5 \mathrm{c}$; 12 at price of $10 \mathrm{c}$; others are net prices and I have but few of them. Orders under $\$ 3.00$, if you want them insured send 5c for insurance. Sums under $\$ 1.00$ you may remit in unused $1 \mathrm{c}$ or $2 \mathrm{c}$ stamps. All bulbs semesan treated. Customers tell me my bulbs are so strong they have to put heavy weights on them to hold them down until they root. If out of a variety will substitute a kind as good or better unless instructed not to do so. I give extra value when substituting. Everything on this list sent prepaid.

\section{GLADIOLI}

Kunderd Rufiled

*Blanche Bollinger-L $8 \mathrm{c}$, IN 5c, $\mathrm{s} 3 \mathrm{c}$, Blbts. 25 for 10c. $/ \mathrm{AN}$

*Bre Ribbon-L 10c, M 7c, S 5c, Blbts. 25 for $15 \mathrm{c}$.

Crinkles-Dark pink, L $8 \mathrm{c}$.

Don Juagn-L 10c, Blbts. 25 for $75 \mathrm{c}$.

*E. J. Shaylor-Pink, L 5c, M 3c, S 25 for $15 \mathrm{c}$.

J. Ogden Armour-Bright coral, salmon red. L $15 \mathrm{c}$.

*Lilac Glory-L 8c.

Masterpiece-L $8 \mathrm{c}$.

Neoga-L $5 \mathrm{c}, \mathrm{M} 3 \mathrm{c}, \mathrm{S} 10$ for $20 \mathrm{c}$.

*Pink Cloud-L 10c, M 7c, S 4c, Blbts. 25 for $25 \mathrm{c}$.

Rosalind-L $8 \mathrm{c}$.

*Sulphur Frills-L 5c, M 3c, 10 for 15c, Blbts. 10 for $10 \mathrm{c}$.

Golden Frills-L $25 \mathrm{c}$.

Battle Creek-L 6c, deep blue.

\section{Kunderd Plain Petal}

Cardinal Prince-Cardinal red. L 10c, M $7 \mathrm{c}$.

Cherry Rose-L $15 \mathrm{c}$.

Dainty (Lascinated) $-\mathrm{L}$ 15c.

Giant Myríle-L 6c.

*Gene Stratton Porter-L 8c, M 5c, S 11 for $20 \mathrm{c}$, Blbts. 25 for $10 \mathrm{c}$.

*Lavender Glow-L 10c, iM 7c, S 10 for 20c, Blbts. 10 for $10 \mathrm{c}$.

*Rajah-Dark red. L 12c, M 8c.

Red Diamond-L $5 \mathrm{c}$.

Robert J. Kunderd-Fine red. L 10c. 
*Queen of Orange-Big range red. L 15c, M Sc, S 5c, Blbts. 10 for $10 \mathrm{c}$.

Lavender Pride-L $10 \mathrm{c}$.

Copper Bronze-Large bloom. L 10c, M 7c, Blbts. 10 for 10c.

E. B. Williamson-Large purple. L 5c.

White Butterfly-L $5 c$.

Mrs. J. C. Bruggan-L 10c, S 4c, Blbts. 10 for $10 \mathrm{c}$.

Show Hlower-Red, white throat. L 10c.

\section{Dieners Varieties}

*Bubbles-Flesh color. Beauty. L 10c, M 7c, S 4c, Blbts. 10 for $10 \mathrm{c}$. Beatriz Michelena-L 10c.

California Giant-Big blue. L 20c, M 15c, S 10c, Blbts. 10 for $10 \mathrm{c}$. Emile Ash-Ruffled white. L 12c.

*Genevieve Bothin-Blooming size 5c. Smoky blue.

*Gladys Plath-Big purple-blue blocm size 5c.

Dr. Bennett-Red. L $10 \mathrm{c}, \mathrm{M} 7 \mathrm{c}$.

*Jack London-L 5c.

*J. Merle Coulter-Deep red fleck throat. L 8c, M ə̃c, Blbts. 25 for $10 \mathrm{c}$.

J. R. Walsh-Red bordered pink. L 8 c.

Martha Fenekes-Light blue. L $15 \mathrm{c}$.

Hoonbeam-White and cream. L $10 \mathrm{c}$.

Sausalito-L $12 \mathrm{c}$.

Waikiki-Pink tinted. ruffled. L 10c. M 7c. S 5c, Blbts. 10 for $10 \mathrm{c}$.

Wm. Kent-Ruffled beauty. L $15 \mathrm{c}$.

Yosemite-7 ft. L $6 \mathrm{c}$.

J. K. Ormsby-L $5 \mathrm{c}$.

\section{Miscellaneous}

*Aristocrat (Bill)—Saffron pink, ruffled. L 10c, M 5c, S 3c, Blbts. 10 for $10 \mathrm{c}$.

*A. V. Bonce (Bill)-Burnt orange. L 10c, M 7c, S 4c, Blbts. 10 ior $10 \mathrm{c}$.

*Bengal 'iger-L 5c. All colors.

Betty Bunce-Lilac and phlox purple. L 10c, M 7c.

Break-0-Day-Pink. L 5c, M 3c, Blbts. 25 for $10 \mathrm{c}$.

Rlue Torch-L 15c, Blbts. 10 for $10 \mathrm{c}$.

Chamelecn-All colors. L 6c, Blbts. 25 for $10 \mathrm{c}$.

Chas. Berther (French) - Light blue, white throat. L 15c, Blbts. 10 for $10 \mathrm{c}$.

*Del Rosa (Burbank)-Large scarlet, light throat. L 6c, M 4c, Blbts. 15 for $10 \mathrm{c}$.

*Del Cra (Burbank)—Large salmon, and yellow. L 6c, M 4c, Blibts. 15 for $10 \mathrm{c}$.

Florence (French)-Fine blue blooming size, 10c.

*Ft. Kantzleiter-Imported big red. L $10 \mathrm{c}$.

*H. Kantzleiter-Imported big red. L $10 \mathrm{c}$.

Gladdy Boy-Large Grenadine Pink. Frilled. L 15c, M 8c.

Gloriana-Rich salmon. L $20 \mathrm{c}$.

Giant Nymph-Large pink. L $6 \mathrm{c}$. 
Golden Dream-L 25c, Blbts. 10 for $20 \mathrm{c}$.

Gild Eaglc-L 15c, Blbts. 10 for 20c.

*High Nye-Tall, smoky. L 10c, M 5c, S 3c, Blbts. 15 for $10 \mathrm{c}$.

*Numdinger-L $8 \mathrm{c}, \mathrm{M} 5 \mathrm{c}, \mathrm{S} 2 \mathrm{c}$, Blbts. 25 for $10 \mathrm{c}$.

Jean Terry (Australia) $\longrightarrow \mathrm{L} 20 \mathrm{c}$.

*Jay's Delight-Large dark blue, yellow throated. Extra choice.

L 15c, M 10c, S 5c, Blibts. 10 for $15 \mathrm{c}$.

Jay's Pink-Large fine pink, extra. L 15c, M 10c, S 6c, Blbts.

10 for $15 \mathrm{c}$.

Kircholi-Violet. L $15 \mathrm{c}$, M $10 \mathrm{c}$.

*Kokoma-Blccm size, smooky, 5c, Blbts. 25 for $10 \mathrm{c}$.

*Los Angeles-Cut and come, pink. L 5c, M 3c, S 25 for 20c, Blbts.

$15 \mathrm{c}$ per 100 .

Louis Hemon (French)-Oddest of all. Bloom size, 20c.

Luther Burbank (Burbank)-Beauty. L $70 \mathrm{c}$.

*Kiva (Burbank)-Fine salmon. Bloom size, 8c.

*Lilac Wonder (imported) - L 8c, M 6c.

Marnin-Dark orange. IL 7c.

M. II. Sully (French beauty)-White, red center. L 15c.

*Maine-Large white. L $5 \mathrm{c}$.

Narretta-Orange. L $5 \mathrm{c}$.

Nimrod (Australia)-Large dark red. L 15c.

*Prince of India-Many colors. L 10c, M 7c, S 5c.

Pride of Wanakah-Beauty, dark lavender. L $10 \mathrm{c}, \mathrm{M} 7 \mathrm{c}, \mathrm{S} 4 \mathrm{c}$, Blbts. 10 for $10 \mathrm{c}$.

Quinton-Salmon. L 5c.

Saraband-Dark violet. L $20 \mathrm{c}$.

Sappho-Smoky. L 12c.

Sunkissed Fairy-Yellow and pink, bloom size, 10c.

*Serratta-Good sized serrated petals. L 18c, IM $10 \mathrm{c}$, S 5c.

*Sunnymede-Fine dark prange. L 5c, M 3c, S 2c, Blbts. 25 for 10c.

Sheilas Sister-Salmon striped. L $6 c$.

Rose Mulberry-Large improved. Rose Ash. L 15c, M $10 \mathrm{c}, \mathrm{S} 5 \mathrm{c}$, Blbts. 10 for $15 \mathrm{c}$.

Sovereign-Large dark blue. L 12c.

*Tiniany-Fine white. L 5c.

Titan (Australia)-Large, pink. L 20c.

Victor (Australia)-Large red, white throat. L $40 \mathrm{c}$.

*Ethelyn-Large, deep orange. L 8c, M 5c, Blibts. 10 for $10 \mathrm{c}$.

*La Verne-Yellow, tinted, pink. L 6c, iN 4c, S 2c, Blibts. 25 for 10c.

Ibosemary-White, speckled pink, beauty. L 6c, M 4c, S 2c, Blbts. 25 for $10 \mathrm{c}$.

Pelican-One of the odd ones. L 6c, M 4c, S 2c, Blbts. 15 for $10 \mathrm{c}$.

Purest of All-The best white. L 16c, M 12c, Blbts. 10 for $10 \mathrm{c}$.

Smoky-Best of all smokies. L $15 \mathrm{c}$.

Giant Blue-L 15c, M 10c, Blbts. 10 for $15 \mathrm{c}$.

Smoky Jay-Fine, large smoky. L 15c.

Pompeii (French)-Blue. L 10c.

Idamae-Light yellow. L 8c, M 5c, Blbts. 25 for $10 \mathrm{c}$.

Frorest Sprite-L 35c. 
Magnolia-Large, pure white. L 15c.

Jay's Beauty-Large, dark pink, white throat. Bloom size, 15c.

The Flirt-Bloom size, 10c.

Just a few pick ups and lost labels. I $35 \mathrm{c}$ doz.

\section{DAHLIAS}

I have over 100 kinds in too small quantities to list. Send list of wants and I will quote prices. Most of them are the good ones.

\section{Show Dahlias}

Copper Bronze-Quilled petals. No other Dahlia has this shade, $25 \mathrm{c}$.

Silver Quartas-Deep lavender. Each petal has an inside petal. The only one in the world of this form, $40 \mathrm{c}$.

Bufi Ball-Round, buff, fine, $15 \mathrm{c}$.

\section{Decorative}

Chas Stratton-Pink, light center, large, $30 \mathrm{c}$.

Copper King (Big one)-Fine, 50c.

Elsie Daniels-Large pink, white center, $30 \mathrm{c}$.

Elite Glory (Big one)-Dark red, $\$ 1.00$.

E. L. Bedford-Large lavender, silver tips, $50 \mathrm{c}$.

Elenor Vandeveer (Big one)-Lavender, $50 \mathrm{c}$.

Ethel Mower-Big delicate light pink, $25 \mathrm{c}$.

Grizzly-Big dark red, extra free bloomer, $40 \mathrm{c}$.

Golden Dream-Always in bloom. $15 \mathrm{c}$.

Margaret Woodrow Wilson (Big one)-Always in bloom, $\$ 1.00$.

Pearl White-Fine bloomer. 20c.

Polar Bear-Fine white. 20c.

John Lewis Childs-Yellow, heavy striped red, free bloomer, $35 \mathrm{c}$. Pride of California-Deep red, fine, $20 \mathrm{c}$.

Creamy Blue-Large, always in bloom, extra good, $25 \mathrm{c}$.

Sagamore-Big dark orange, fine free bloomer, $50 \mathrm{c}$.

Variegated Aster-New type, Needle pointed petals, pink and red, $20 \mathrm{c}$.

\section{Cactus Dahlias}

Brogan-Large dark red, fine, $50 \mathrm{c}$.

King Solomon-Dark yellow, always in bloom, large, $50 \mathrm{c}$.

Rollo Boy-Extra large free bloomer. 50c.

Sunkissed-Salmon, yellow center, fine free bloom $\approx$, $30 \mathrm{c}$.

\section{Peony Dahlia}

Black Lady-Black red, 25c.

White City-Large snow white, pointed petals, $20 \mathrm{c}$,

\section{IRIS}

12 fine mixed, $50 \mathrm{c}, 130$ fine mixed, $\$ 1.00$.

20 , no two alike, $\$ 1.00,40$, no two alike, $\$ 2.00$.

14 named and labeled, kinds all different, $\$ 1.00$.

If you are interested in named kinds send for list of 100 kinds.

\section{J. C. JAY, OZARK VIEW FLOWER FARM}

